\title{
NanoCartography: Mathematics of Crystal Orientation in Double Tilt Holders
}

Kevin Fiedler ${ }^{1}$ and Matthew Olszta ${ }^{2}$

${ }^{1}$ Washington State University, Richland, Washington, United States, ${ }^{2}$ Pacific Northwest National Laboratory, Richland, Washington, United States

Understanding the orientation of a crystal in an electron microscope is crucial for rapid navigation to features of interest. With the increasing specialization of microscope techniques, the cost of instrumentation time has becomes especially precious. In this work, the crystal is treated an object in Cartesian space that can be oriented using multiplication of rotation matrices. Particular care is required for the case of double tilt stages because one axis of rotation is beholden to the other; that is the rotations are not independent so the order in which they are applied mathematically is critical. Once the orientation of the sample has been determined, two applications of the mathematics are investigated in this work. The first was a tip/tilt diagram which converts the geometric object in Cartesian space into tip/tilt coordinates for the double tilt holder. This diagram allows a user to know exactly where to locate additional poles so that they can be rapidly aligned for further study, such as capturing diffraction patterns. The second was calculating the misorientation between two crystals. In this case, the rotation matrix that is required to move from one orientation to another was computed. This formulation allows for deeper understanding of a sample across interfaces or grain boundaries where two different crystals interact.

To construct a tip/tilt diagram, the mathematics follows the regular workflow of analyzing a sample. Specifically, it is split into three steps: loading, crystal alignment, and stage alignment. The first step, Rloading, represents the orientation of the sample and holder as it is inserted into the microscope. The

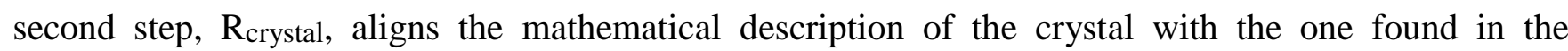
microscope sample. The last step, $\mathrm{R}_{\text {stage, }}$ puts the pole at the location corresponding to the known tip/tilt coordinates observed. Taken together, the multiplication of these matrices yields an overall rotation matrix, $\mathrm{R}_{\text {total }}=\mathrm{R}_{\text {stage }} \mathrm{R}_{\text {crystal }} \mathrm{R}_{\text {loading, }}$, that contains all the orientation information about the crystal as it is situated in the microscope in the present configuration. More importantly, it also contains the information about the crystal at any other configuration allowing for the creation of the tip/tilt diagram. To accomplish this, the Cartesian representation of the crystal found from $\mathrm{R}_{\text {total }}$ was converted to tip/tilt coordinates and the locations of poles were plotted. Once these poles have been plotted a microscopist can immediately move to any desired pole allowed by the sample orientation.

Given two crystals with different orientations, the amount of misalignment can be quantified through a rotation matrix. To find the rotation matrix that takes one crystal to the orientation of a second three independent poles for a crystal are first determined from the tip/tilt diagram. These poles are converted from tip/tilt coordinates to Cartesian coordinates. Using these coordinates, the principal axes of the crystal are determined and rotated to a known reference state (usually aligning a principal axis with the

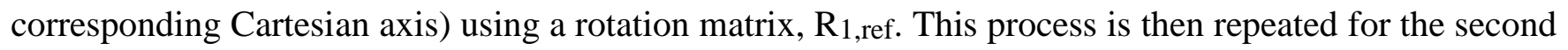
crystal to find its rotation matrix to the reference state, $\mathrm{R}_{2}$,ref. To convert from the first orientation to the second, the rotation matrix rotates from the first orientation to the reference state and then from the reference state to the second orientation, $\mathrm{R}_{1,2}=\mathrm{R}_{1, \text { ref }} \mathrm{R}_{\mathrm{ref}, 2}$. This overall rotation matrix then yields the axis of rotation and angle required to go from one orientation to the other. This angle is commonly called the misorientation angle and gives critical information about the relationship between different crystals within 
a sample. These tools establish the basics of NanoCartogrpahy whereby users can quickly and efficiently navigate the world of electron microscope samples.

\section{References}

Cautaerts, N., R. Delville, and D. Schryvers, ALPHABETA: a dedicated open-source tool for calculating TEM stage tilt angles. Journal of Microscopy, 2018. 273.

Cole, I. R. "Modelling CPV", Ph.D. thesis (Loughborough University), 2015.

Liu, Q., A simple method for determining orientation and misorientation of the cubic crystal specimen. Journal of Applied Crystallography, 1994. 27(5): p. 755-761.

Liu, Q., A simple and rapid method for determining orientations and misorientations of crystalline specimens in TEM. Ultramicroscopy, 1995. 60(1): p. 81-89.

Qing, L., An equation to determine the practical tilt angle of a double-tilt specimen holder and its application to transmission electron microscopy. Micron and Microscopica Acta, 1989. 20(3): p. 261-264. Qing, L., M. Qing-Chang, and H. Bande, Calculation of tilt angles for crystal specimen orientation adjustment using double-tilt and tilt-rotate holders. Micron and Microscopica Acta, 1989. 20(3): p. 255259. 